\title{
Infants' perception of depth from cast shadows
}

\author{
ALBERT YONAS \\ University of Minnesota, Minneapolis, Minnesota \\ and \\ CARL E. GRANRUD \\ University of Northern Colorado, Greeley, Colorado
}

\begin{abstract}
Five- and 7-month-old infants viewed displays in which cast shadows provided information that two objects were at different distances. The 7-month-olds reached preferentially for the apparently nearer object under monocular-viewing conditions but exhibited no reaching preference under binocularviewing conditions. These results indicate that 7-month-old infants perceive depth on the basis of cast shadows. The 5-month-olds did not reach preferentially for the apparently nearer object and, therefore, exhibited no evidence of sensitivity to cast shadows as depth information. In a second experiment, 5month-olds reached preferentially for the nearer of two objects that were similar to those used in the first experiment but were positioned at different distances from the infant. This result indicated that 5-month-olds have the motor skills and motivation necessary to exhibit a reaching preference under the conditions of this study. The results are consistent with the hypothesis that depth perception based on cast shadows first appears between 5 and 7 months of age.
\end{abstract}

In the 15th century, Leonardo da Vinci described a set of techniques that painters could use to portray threedimensionality on a flat canvas. In his description, he distinguished between two types of shadows: attached shadows and cast shadows. Attached shadows occur when an object's shadow is visible on that same object, such as shading on a ball. A cast shadow occurs when the shadow of one object is seen on a different object or surface, such as a ball's shadow on the ground. Leonardo noted that attached shadows provide information for surface relief, whereas cast shadows indicate a separation in space between objects (Woodworth, 1938).

More recently, computer vision researchers have made detailed descriptions of the information provided by shadows and shading for the perception of object shape and spatial layout (e.g., Horn, 1975; Waltz, 1975), and perception researchers have studied how the visual system uses this information in object and space perception. For example, Koenderink, van Doorn, and Kappers $(1992,1996)$ have shown that shading (i.e., attached shadows) provides a rich source of information used by the adult visual system for perceiving local surface orientation and three-

\footnotetext{
This research was supported by Grant HD-07151 provided to the Minnesota Cognitive Science Center by NICHD and was first presented at the 2001 meeting of the Society for Research in Child Development. The authors thank Amelia Alexander, Jill Kawaguchi, Nou Yang, Melissa Farr, Ann O'Connor, and Allyson Candee for their assistance in conducting the study and Scott Johnson, M. A. Schmuckler, Bruce Schneider, and Allison Sekuler for their helpful comments regarding the manuscript. Correspondence concerning this article may be sent to A. Yonas, Institute of Child Development, 51 E. River Road, Minneapolis, MN 55455 (e-mail: yonas@umn.edu).
}

dimensional object shape. In addition, Kersten, Knill, Mamassian, and Bülthoff (1996) have demonstrated that adults use cast shadow information to perceive objects' distances and movements in three-dimensional space.

Several studies have been done in which the development of space perception based on shading and shadows has been investigated by testing children's and infants' sensitivity to these depth cues. Benson and Yonas (1973) found that 3-year-old children perceive surface relief on the basis of attached shadows. The children in their study discriminated bumps and dents that were specified by shading. In a subsequent study, Yonas, Goldsmith, and Hallstrom (1978) found that 3-year-old children can judge objects' relative distances on the basis of cast shadows. In the first study of infants' sensitivity to depth information provided by shading, Granrud, Yonas, and Opland (1985) found that 7-month-old infants perceive surface relief on the basis of shading but found no evidence of this ability in 5-month-old infants. This developmental pattern was similar to that found in studies of infants' sensitivity to other pictorial depth cues, including linear perspective (Arterberry, Bensen, \& Yonas, 1991; Yonas, Cleaves, \& Pettersen, 1978; Yonas, Granrud, Arterberry, \& Hanson, 1986), interposition (Granrud \& Yonas, 1984), line junctions (Yonas \& Arterberry, 1994), familiar size (Granrud, Haake, \& Yonas, 1985; Yonas, Pettersen, \& Granrud, 1982), relative size (Yonas, Granrud, \& Pettersen, 1985), and surface contours (Sen, Yonas, \& Knill, 2001).

It has remained unknown whether infants perceive depth on the basis of cast shadows or whether sensitivity to cast shadows as depth information emerges at the same point in development as sensitivity to depth based on shading or other pictorial depth cues. The present study 
had two goals. The first was to determine whether infants perceive depth on the basis of cast shadows. The second was to investigate the development of this ability.

\section{EXPERIMENT 1}

In Experiment 1, 5- and 7-month-old infants viewed computer-generated displays that contained cast shadow information for relative distance. Figure 1 shows one of the two displays that were used. Each display depicted two objects, each casting a virtual shadow onto a background surface. The virtual shadow was shifted $2.3 \mathrm{~cm}$ to the left, relative to one object (the object on the right in Figure 1). This displacement between the object and its shadow provided information that the object was separated in space from the background surface. The virtual shadow was shifted $0.5 \mathrm{~cm}$ to the left, relative to the other object (the object on the left in Figure 1). The proximity between this object and its shadow provided information that the object was close to the background surface. The displays were actually flat surfaces, and the two objects were equally distant from the research participant. In a pilot study, adults and 3-year-old children reliably reported that the object whose shadow was displaced $2.3 \mathrm{~cm}$ appeared to be nearer than the object whose shadow was displaced $0.5 \mathrm{~cm}$. Infants viewed two displays, one at a time, on alternating trials. In one display, the apparently nearer object was on the left; in the other display, the apparently nearer object was on the right.

Infants' reaching behavior was measured. Previous studies have shown that 5- and 7-month-old infants reach preferentially for the nearer of two objects (e.g., Granrud, Yonas, \& Pettersen, 1984). On the basis of these findings, infants would be expected to reach preferentially for the apparently nearer of two objects whose relative distances were specified by cast shadows, if they could perceive distance on the basis of cast shadow information.

To control for stimulus variables other than perceived depth, two conditions were included in the study: a monocular-viewing condition and a binocular-viewing condition. The infant wore eyeglasses throughout the experiment. In the monocular condition, an opaque lens blocked the view to one eye; in the binocular condition, the displays were visible to both eyes. If infants reach preferentially for the apparently nearer object on the basis of perceived depth, a reaching preference would be expected in the monocular-viewing condition only. In the binocular condition, binocular disparity and convergence provided information that the two objects were equidistant. Several studies on infant depth perception have shown that binocular information for equal distances overpowers conflicting pictorial cues that specify depth (Granrud, Haake, \& Yonas, 1985; Granrud \& Yonas, 1984; Granrud, Yonas, \& Opland, 1985; Yonas, Cleaves, \& Pettersen, 1978; Yonas et al., 1986; Yonas et al., 1985). If preferential reaching is based on perceived depth, no reaching preference would be expected in the binocular-viewing condition. However, if infants reached preferentially for one of the objects on the basis of stimulus variables other than perceived depth,

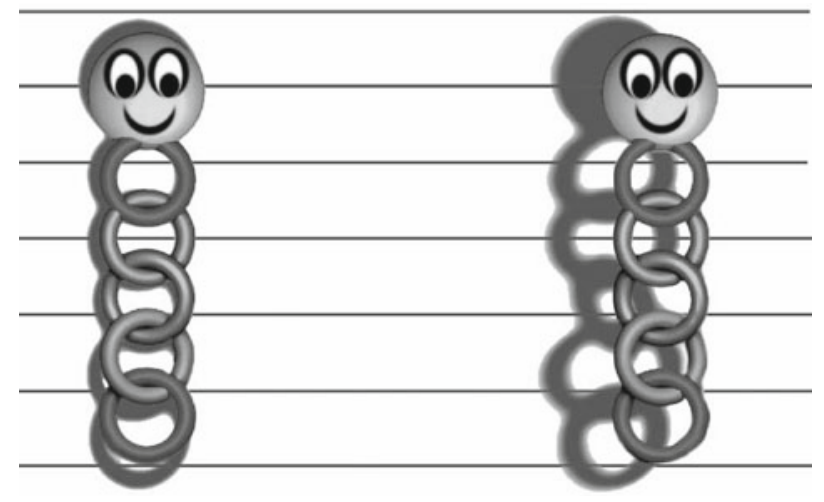

Figure 1. Display used in Experiment 1. The virtual shadows created a depth illusion. Adults and children consistently reported that the object on the right appeared to be nearer than the object on the left.

similar reaching preferences would be expected in the two viewing conditions. For example, the overall width of the object plus its shadow and the overall amount of contrast are greater on the side of the apparently nearer object than on the side of the apparently farther object. Since all nondepth stimulus variables, such as overall width and contrast, were present in both viewing conditions, a reaching preference based on any of these variables would be expected in both the monocular- and the binocular-viewing conditions.

\section{Method}

Participants. The sample consisted of 43 five-month-old infants (23 males and 20 females, $20-22$ weeks in age, mean age $=$ 21.1 weeks) and 42 seven-month-old infants (16 males and $26 \mathrm{fe}-$ males, 27-31 weeks in age, mean age $=29.5$ weeks). An additional 21 five-month-olds and 18 seven-month-olds were tested but were excluded from the sample; 3 five-month-olds and 4 seven-montholds were excluded because of experimenter error, and 18 fivemonth-olds and 14 seven-month-olds were excluded for failure to meet the inclusion criterion of at least six reaches in each viewing condition (in each case, due to fussiness). The infants were recruited using county birth records. The sample consisted of infants whose parents responded to a letter and volunteered to participate with their infant. The parents gave informed consent before testing began. The infants came primarily from white middle-class families.

Apparatus. Two displays were presented, one at a time. Each display was a computer-generated image depicting two identical objects designed to resemble toy rattles. Each object measured $14 \mathrm{~cm}$ in height and $4 \mathrm{~cm}$ in width and consisted of a yellow smiling face and a body of chain-linked purple, orange, blue, and green circles (see Figure 1). The two objects were separated by $13 \mathrm{~cm}$ at their nearest points. The objects were presented on a background of seven blue horizontal lines spaced $2.5 \mathrm{~cm}$ apart. These lines were included in the display to provide information that the background surface was oriented in a frontoparallel plane.

Each object cast a virtual shadow. The virtual shadow was shifted $2.3 \mathrm{~cm}$ to the left relative to one object. This object was referred to as the apparently nearer object. The virtual shadow was shifted $0.5 \mathrm{~cm}$ to the left relative to the other object. This object was referred to as the apparently farther object. The apparently nearer object was on the left in one display and on the right in the other display. The two displays were identical in all other respects. A lamp containing a $40-\mathrm{W}$ incandescent bulb was visible immediately to the right of the 
display. The lamp was positioned in such a way that it would have created the virtual shadows had the objects been positioned at different distances from the background surface. The 40-W lamp was the principal light source in the testing room (a video monitor emitted a small amount of additional light).

The displays were presented in a vertical orientation, perpendicular to the infants' line of sight. Each display was $28 \mathrm{~cm}$ wide and $21.5 \mathrm{~cm}$ high and was mounted on a rigid, white foam-core board, $46 \mathrm{~cm}$ wide and $25 \mathrm{~cm}$ high. The foam-core board was attached to a spring that allowed approximately $10 \mathrm{~cm}$ of vertical and horizontal movement. This bouncing movement was introduced to attract and maintain the infants' attention. A wind chime was also placed behind the apparatus to attract attention.

The apparatus rested on a table. The infant sat on a parent's lap in front of the display. The parent was instructed to hold the infant around the waist, leaving the arms and hands free to move. To prevent the parent from biasing the results, he or she was kept blind as to the specific hypotheses of the experiment and was not informed of the viewing condition conducted on any given trial. The display was positioned out of reach before each trial and was moved in a sliding motion toward the infant at the beginning of each trial. The infant's view of the display was blocked between trials by a maroon screen, $32 \mathrm{~cm}$ wide and $45.3 \mathrm{~cm}$ high.

The infant wore eyeglass frames throughout the experiment. The frames contained no lens on the right side. An opaque plastic lens on the left side of the frames could be rotated downward to block the view to the left eye in the monocular-viewing condition or rotated upward to allow binocular viewing.

Each infant's experimental session was recorded by a video camera mounted in the ceiling above the infant. The top of the display was marked with lines that divided the display into three regions that were used for coding the infants' reaches. A 7-cm-wide region was centered over each object. Reaches to these regions were coded as reaches directed toward the objects. Reaches to the $10-\mathrm{cm}$ region separating these regions were coded as reaches toward the middle of the display.

Procedure. Each infant participated in two conditions: the monocular-viewing condition and the binocular-viewing condition. The conditions were presented in counterbalanced order, with alternating infants receiving the monocular or binocular condition first.

Before each trial, the display was positioned beyond the infant's reach, and the maroon occluder blocked the infant's view of the display. At the beginning of each trial, the occluder was removed, and the display was moved in a sliding motion along the table toward the infant. If necessary, the experimenter touched the display on the midline of its top edge, to initiate a slight bouncing movement, and tapped the wind chime to attract the infant's attention. The trial ended when the infant's hand first contacted the display. The display was then moved out of reach, and the occluder was put into place to block the infant's view while a new display was put into position.

Eight trials were completed in the first viewing condition. The infant then received a short break. Eight trials were then conducted in the second viewing condition. The left-right position of the apparently nearer object was reversed on alternating trials. Left-right position on the first trial was counterbalanced across infants. After the infant completed both viewing conditions, an additional 8-trial block was conducted in each viewing condition if the infant appeared to remain interested in the task. The testing session ended when the infant was judged to have lost interest or when 16 trials had been completed in each condition. Infants were excluded from the sample if they completed fewer than 6 trials in either viewing condition.

The infants' reaches were scored from videotape by an observer who was blind to viewing condition and to the left-right position of the apparently nearer object. The infant's image was masked on the video screen. Only the top edge of the display and the infant's hands were visible on the video monitor. This was done to prevent the observer from seeing whether the infant was participating in the monocular- or the binocular-viewing condition on any given trial. The infants' reaches were categorized as being directed to the left, right, or middle of the display or to both the left and the right simultaneously with two hands. Reaches to the left or the right were later recoded as reaches to the apparently nearer and apparently farther objects.

Only reaches toward the apparently nearer and apparently farther objects were included in analyses of the data. The percentage of reaches directed toward the apparently nearer object, out of the total number of reaches toward the apparently nearer and farther objects, was computed for each infant. This percentage was the dependent variable used in all the analyses. Reaches toward the middle of the display and toward both objects simultaneously were uninformative regarding depth perception from cast shadows and were omitted from the data used in the analyses. This allowed the analyses to compare directly reaches to the apparently nearer object versus reaches to the apparently farther object. If the infants reached more often for the apparently nearer object than for the apparently farther object, in the monocular condition only, it would indicate depth perception based on cast shadows, regardless of how often the infants reached for both objects simultaneously or for the middle of the display.

\section{Results}

Table 1 shows the mean number of trials completed and the mean number of reaches directed toward the apparently nearer object, the apparently farther object, and the middle of the display or both objects simultaneously for each age group in each viewing condition. Table 2 shows the mean percentages of reaches that were directed toward the apparently nearer object in each condition, computed after reaches to both objects and to the middle of the display were excluded from the data. All the analyses were conducted using the means shown in Table 2.

Planned paired-samples $t$ tests were conducted to compare the means from the monocular and binocular conditions

Table 1

Mean Number of Trials Completed and Mean Number of Reaches Toward the Apparently Nearer Object, Apparently Farther Object, and Middle or Both Objects in Each Viewing Condition

\begin{tabular}{|c|c|c|c|c|c|c|c|c|}
\hline \multirow[b]{3}{*}{ Age Group } & \multicolumn{8}{|c|}{ Viewing Condition } \\
\hline & \multicolumn{4}{|c|}{ Monocular } & \multicolumn{4}{|c|}{ Binocular } \\
\hline & Trials & Near & Far & Middle/Both & Trials & Near & Far & Middle/Both \\
\hline \multicolumn{9}{|l|}{5 months } \\
\hline Mean & 7.79 & 2.86 & 2.91 & 2.02 & 7.97 & 2.74 & 2.72 & 2.51 \\
\hline$S D$ & 0.56 & 1.30 & 1.39 & 1.57 & 0.74 & 1.42 & 1.37 & 1.62 \\
\hline \multicolumn{9}{|l|}{7 months } \\
\hline Mean & 10.86 & 5.43 & 4.12 & 1.31 & 11.09 & 4.95 & 4.81 & 1.33 \\
\hline$S D$ & 3.75 & 2.13 & 2.48 & 1.47 & 3.07 & 2.21 & 2.00 & 1.60 \\
\hline
\end{tabular}


Table 2

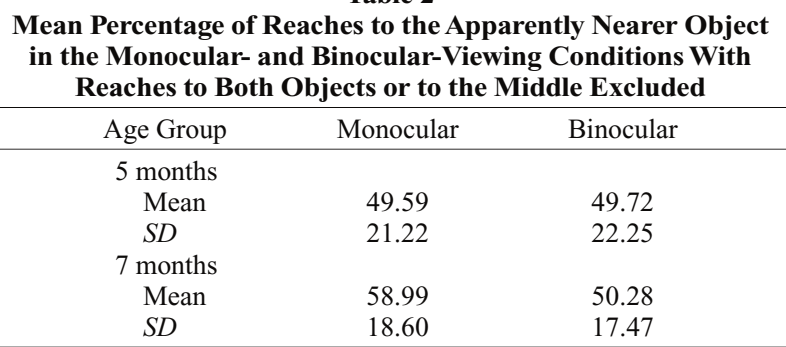

Note-Because some infants completed unequal numbers of trials, the mean percentages differ slightly from the percentages that could be calculated from the mean numbers of reaches to the apparently near and far objects shown in Table 1

within each age group. The 7-month-old infants exhibited a significantly stronger tendency to reach for the apparently nearer object in the monocular condition than in the binocular condition $[t(41)=2.27, p<.05]$. The 5-montholds' means did not differ between the monocular- and the binocular-viewing conditions $[t(42)=-0.03, p>.05]$. Planned one-sample $t$ tests were conducted to compare each age group's mean with $50 \%$ in each viewing condition. The 7-month-olds' mean differed significantly from $50 \%$ in the monocular-viewing condition $[t(41)=3.13, p<$ $.05]$, but not in the binocular-viewing condition $[t(41)=$ $0.10, p>.05]$. The 5-month-olds' means did not differ from $50 \%$ in either the monocular $[t(42)=-0.13, p>.05]$ or the binocular $[t(42)=-0.08, p>.05]$ condition.

Additional analyses revealed that sex and order of the monocular- and binocular-viewing conditions had no significant effects on the infants' reaching behavior and that the 5-month-olds did not exhibit a reaching preference in the early trials that disappeared in the later trials of the experiment.

\section{Discussion}

The results indicate that 7-month-old infants perceive depth on the basis of cast shadows. These infants exhibited a significant reaching preference for the apparently nearer object in the monocular condition and showed no reaching preference in the binocular condition. This pattern of results suggests that the 7-month-old infants' reaching behavior was based on perceived depth. In the monocular condition, in which binocular depth cues for flatness were absent, the 7-month-old infants apparently perceived that the object whose virtual shadow was displaced $2.3 \mathrm{~cm}$ was nearer than the object whose virtual shadow was displaced $0.5 \mathrm{~cm}$. In the binocular condition, these infants apparently perceived that the two objects were equidistant and, therefore, did not reach preferentially for one object. The finding of no reaching preference in the binocular condition indicates that the 7-month-old infants' monocular condition reaching preference was not based on stimulus variables other than perceived depth. Had the infants reached preferentially for one object on the basis of overall display width or contrast, for example, a reaching preference would have been expected in both the monocular and binocular conditions, since these stimulus variables were equated in the two viewing conditions.

The 5-month-old infants did not exhibit a reaching preference for the apparently nearer object. The results, therefore, provided no evidence that 5-month-old infants perceive depth on the basis of cast shadows. However, this experiment did not find conclusive evidence that 5month-old infants cannot perceive depth from cast shadows. Although 5-month-olds reach preferentially for the nearer of two objects when binocular or kinetic depth cues specify relative distance (Granrud, Yonas, \& Pettersen, 1984; Granrud, Yonas, Smith, et al., 1984), it is possible that the 5-month-olds perceived depth in Experiment 1 but lacked the motor abilities or motivation necessary to exhibit a reaching preference under the conditions in this experiment. Experiment 2 was designed to explore this possibility.

\section{EXPERIMENT 2}

In Experiment 2, we investigated whether 5-month-old infants would exhibit a reaching preference for the nearer of two objects under conditions similar to those in Experiment 1. In Experiment 2, 5-month-olds viewed displays identical to those in Experiment 1, with the exception that one object was raised $4 \mathrm{~cm}$ from the background surface and one object was raised $0.5 \mathrm{~cm}$ from the background surface. At these distances from the background, the objects cast shadows similar in size and position to the virtual shadows in the displays used in Experiment 1. Each infant was tested under monocular- and binocular-viewing conditions. In the monocular condition, cast shadows, motion parallax, and accommodation provided information for the objects' relative distances. In the binocular condition, binocular disparity and convergence were available, in addition to the depth cues present in the monocular condition. If 5-month-olds exhibited a reaching preference in Experiment 2, it would suggest that these infants had the abilities and motivation necessary to exhibit a reaching preference under the conditions of this study when adequate visual information was available for depth to be perceived. The finding of a reaching preference in Experiment 2 would, therefore, support the conclusion that the 5-month-olds' lack of a reaching preference in Experiment 1 resulted from an inability to perceive depth on the basis of cast shadows.

\section{Method}

Participants. The sample consisted of 12 five-month-old infants ( 6 males and 6 females, 20-22 weeks of age, mean age = 21.3 weeks). Eight additional 5-month-old infants were tested but were excluded from the sample for not meeting the inclusion criterion of six reaches per condition (in each case, due to fussiness).

Apparatus. The apparatus used in Experiment 2 was identical to that used in Experiment 1, with two exceptions. First, in each of the two displays used in Experiment 2, one object (the near object) was raised $4 \mathrm{~cm}$ from the background surface toward the infant, and one object (the far object) was raised $0.5 \mathrm{~cm}$ from the background surface. The near object was on the left in one display and on the right in the other display. Second, the displays used in Experiment 2 
did not contain printed virtual shadows, as they did in Experiment 1. Instead, the raised objects in the displays, which were illuminated by the same 40-W lamp as that used in Experiment 1, cast real shadows onto the background surface that were similar in size and position to the virtual shadows in the displays used in Experiment 1.

Procedure. The procedure used in Experiment 2 was identical to that used in Experiment 1, with three exceptions. First, all of the infants received six trials in each viewing condition. Second, some of the infants wore an adhesive eye patch in the monocular condition, instead of eyeglasses. Third, in the coding of the infants' reaches from the videotaped record of the experiment, a reach was scored when any part of the infant's hand crossed a plane $4 \mathrm{~cm}$ in front of, and parallel to, the background surface. Reaches were coded as being directed to the near object, the far object, the middle of the display, or both objects simultaneously, following the same procedures as those used in Experiment 1. As in Experiment 1, only reaches to the near and far objects were included in analyses of the data; reaches for the middle of the display or for both objects were omitted from the analyses.

\section{Results}

Table 3 shows the mean numbers of reaches directed toward the near object, the far object, and the middle of the display or both objects simultaneously in each viewing condition. With reaches for the middle of the display and for both objects simultaneously excluded from the data, the mean percentages of reaches directed toward the near object were $76.67 \%(S D=21.84)$ in the binocular condition and $65.14 \%(S D=21.19)$ in the monocular condition. (These mean percentages differ slightly from the percentages that could be calculated from Table 3, because some infants had unequal numbers of total reaches after middle/both reaches were excluded.) Planned one-sample $t$ tests were conducted to compare the mean in each viewing condition with $50 \%$. The infants exhibited significant reaching preferences for the near object in the binocular$[t(11)=4.23, p<.05]$ and monocular- $[t(11)=2.47$, $p<.05]$ viewing conditions. A planned paired-samples $t$ test showed no significant difference between the infants' reaching preferences in the binocular- and monocularviewing conditions $[t(11)=1.24, p>.05]$.

\section{Discussion}

The results of Experiment 2 indicate that 5-month-old infants reach preferentially for the nearer of two objects when motion parallax and accommodation (in the monocular condition) and binocular disparity and convergence (in the binocular condition), in addition to cast shadows, provide information on the objects' relative distances.

Table 3

Mean Number of Reaches Toward the Apparently Nearer Object, Apparently Farther Object, and Middle or Both Objects in Each Viewing Condition in Experiment 2

\begin{tabular}{cccc}
\hline Condition & Near & Far & Middle/Both \\
\hline Monocular & & & \\
Mean & 3.50 & 1.83 & 0.67 \\
SD & 1.24 & 1.03 & 0.78 \\
Binocular & & & \\
Mean & 4.17 & 1.33 & 0.50 \\
SD & 1.27 & 1.23 & 0.90 \\
\hline
\end{tabular}

When adequate depth information is available, 5-monthold infants have the motor skills and motivation necessary to exhibit a consistent reaching preference for the nearer of two objects under conditions nearly identical to those in Experiment 1. The results of Experiment 2, therefore, suggest that the 5-month-olds' behavior in Experiment 1 cannot be attributed to a deficit in the motor skills or motivation necessary to exhibit a reaching preference under the conditions in this study.

\section{GENERAL DISCUSSION}

The results of this study indicate that 7-month-old infants perceive depth from information provided by cast shadows. In the monocular-viewing condition in Experiment 1, 7-month-olds exhibited a significant reaching preference for the apparently nearer of two objects whose relative distances were specified by virtual cast shadows. In the binocular condition in Experiment 1, 7-month-olds exhibited no reaching preference, indicating that their reaching behavior was based on perceived depth, and not on low-level stimulus variables.

This study showed no evidence that 5-month-old infants perceive depth on the basis of cast shadows. These infants exhibited no reaching preference in Experiment 1. In Experiment 2, when two objects, similar to those used in Experiment 1, were positioned at different distances from 5-month-old infants, the infants showed consistent reaching preferences for the nearer of two objects under monocular- and binocular-viewing conditions. This result suggests that 5-month-old infants have the motor abilities and motivation needed to exhibit a reaching preference under the conditions in this study. These results do not demonstrate conclusively that sensitivity to cast shadows as depth information is absent in 5-month-old infants. It is possible that better experimental methods would reveal sensitivity to cast shadows in 5-month-olds. The results suggest, however, that depth perception based on cast shadows may first appear between 5 and 7 months of age.

Although the 7-month-old infants exhibited a significant reaching preference in the monocular-viewing condition in Experiment 1, this preference was weak, and it was less consistent than the reaching preferences exhibited by the 5-month-olds in Experiment 2. The weakness of the 7-month-olds' reaching preference most likely resulted from conflict between the depth cues that were present in the displays used in Experiment 1. Cast shadows specified that the two objects were at different distances. At the same time, in the monocular-viewing condition, motion parallax (generated by the infants' head movements) and accommodation specified that the objects were equidistant. In the binocular condition, binocular disparity and convergence also conflicted with the information provided by cast shadows and specified that the objects were equidistant. When all of the depth cues present provide consistent information for relative distance, as in Experiment 2, 5- and 7-month-old infants consistently exhibit 
strong reaching preferences, directing $75 \%$ or more of their reaches toward the nearer of two objects or the nearer side of a slanted object (e.g., Granrud, Yonas, \& Pettersen, 1984; Yonas, Cleaves, \& Pettersen, 1978). In studies of infants' sensitivity to pictorial depth cues, in which pictorial cues for depth conflict with motion parallax and accommodation cues that specify equidistance, reaching preferences are consistently weaker. Seven-month-old infants typically direct about $60 \%-65 \%$ of their reaches toward the apparently nearer of two objects under monocularviewing conditions in these studies (e.g., Granrud, Haake, \& Yonas, 1985; Granrud \& Yonas, 1984; Granrud, Yonas, \& Opland, 1985; Yonas, Cleaves, \& Pettersen, 1978; Yonas et al., 1986; Yonas et al., 1985). The 7-month-olds' 59\% reaching preference in the present study was, therefore, consistent with previous research in which reaching has been used as a measure of depth perception in infants.

A consistent developmental pattern has been found in several studies in which infants' perception based on shading and shadows has been explored. Granrud, Yonas, and Opland (1985) found that 7-month-old infants perceived surface relief on the basis of shading but found no evidence of this ability in 5-month-olds. In a more recent study by Yonas and O'Donnell (1996), 7-month-old infants attended to luminance boundaries created by changes in reflectance but did not attend to similar luminance boundaries created by shadows. In contrast, 5-month-olds in Yonas and O'Donnell's study paid equal attention to luminance boundaries created by reflectance changes and luminance boundaries created by shadows. It seems likely that the ability to distinguish shadows from reflectance changes is a prerequisite for detecting distance or shape information provided by shadows and shading. For example, the shadow cast by a person's nose onto the person's cheek provides information about the size and shape of the nose. But the shadow must be perceived as a shadow, and not as a facial feature itself, for this information to be detected. Similarly, in Figure 1, the cast shadows must be perceived as shadows, and not as variations in reflectance, to be used as cues for distance. The results of the present study, together with those of Yonas and O'Donnell and of Granrud, Yonas, and Opland, suggest the hypothesis that the ability to distinguish shadows from reflectance changes and the abilities to perceive distance and shape from shadows and shading appear between 5 and 7 months of age. Furthermore, the latter abilities may depend on prior development of the former ability.

The development of sensitivity to the information provided by shading and shadows may be related to the development of a more general perceptual ability: the ability to perceive depth and three-dimensionality from the information available in a single, static retinal image. Studies have shown that 7-month-old infants perceive depth on the basis of the pictorial (i.e., static-monocular) depth cues of linear perspective, interposition, relative size, familiar size, line junctions, and surface contour (Arterberry et al., 1991; Granrud, Haake, \& Yonas, 1985; Granrud \& Yonas, 1984; Sen et al., 2001; Yonas \& Arterberry, 1994; Yonas,
Cleaves, \& Pettersen, 1978; Yonas et al., 1986; Yonas et al., 1985; Yonas et al., 1982). None of these studies showed evidence that 5 -month-olds are sensitive to these pictorial depth cues. Although future research may reveal that 5-month-old infants are sensitive to pictorial depth cues, including cast shadows, the consistent pattern shown in numerous studies suggests that sensitivity to many, or all, pictorial depth cues may first appear between 5 and 7 months of age.

At present, it remains unknown what causes this apparent developmental change in perception. However, several hypotheses can be ruled out on the basis of the data that currently exist. Arterberry et al. (1991), using a habituation-of-looking method, found that 7-month-olds perceive depth from linear perspective but found no evidence of this ability in 5-month-olds. This suggests that differences between 5- and 7-month-olds in responsiveness to pictorial depth cues cannot be attributed to the use of reaching as a measure of depth perception. Arterberry, Yonas, and Bensen (1989) found no differences between 7-month-olds with varying degrees of crawling experience in sensitivity to linear perspective, suggesting that the development of sensitivity to pictorial depth cues is not related to the development of crawling. Finally, Gunderson, Yonas, Sargent, and Grant-Webster's (1993) finding that 7-week-old pigtailed macaque monkeys perceive depth from linear perspective and relative size indicates that the ability to perceive depth from pictorial cues is not acquired on the basis of learned cultural conventions, as has sometimes been suggested (e.g., Deregowski, 1968; Goodman, 1976; Hudson, 1967).

Several other hypotheses remain untested. For example, Sen et al. (2001) proposed that, in the early months of life, infants acquire implicit knowledge about regularities in the environment that makes perception based on pictorial depth cues possible. They may learn, for example, that light comes from above (necessary for perceiving shape from shading) and that multiple linear contours that converge in the retinal image are parallel in the threedimensional world (necessary for perceiving distance from linear perspective). Using this implicit knowledge to perceive depth, however, may depend on the maturation of brain mechanisms that allow the top-down application of knowledge in the interpretation of visual information. Sen et al. hypothesized that descending neural pathways from the temporal lobe to extrastriate areas in the occipital lobe, which allow top-down processing in visual perception, mature and become functional between 5 and 7 months of age in human infants. A testable prediction implied by this hypothesis is that many perceptual abilities that involve using knowledge to interpret ambiguous visual information, in addition to those used in depth perception, first appear in infants between 5 and 7 months of age. An alternative possibility is that the implicit knowledge needed for depth perception from pictorial cues was built into the visual system during our evolutionary history and is not acquired through experience. Further research, such as animal studies in which early visual experience is care- 
fully controlled, will be needed to test these hypotheses and to identify the specific brain mechanisms involved in the perceiving of depth from pictorial cues.

\section{REFERENCES}

Arterberry, M. E., Bensen, A. S., \& Yonas, A. (1991). Infants' responsiveness to static-monocular depth information: A recovery from habituation approach. Infant Behavior \& Development, 14, 241-251.

Arterberry, M. [E.], Yonas, A., \& Bensen, A. S. (1989). Self-produced locomotion and the development of responsiveness to linear perspective and texture gradients. Developmental Psychology, 25, 976-982.

Benson, C., \& Yonas, A. (1973). Development of sensitivity to static pictorial depth information. Perception \& Psychophysics, 13, 361-366.

DeregowsKi, J. B. (1968). Difficulties in pictorial depth perception in Africa. British Journal of Psychology, 59, 195-204.

Goodman, N. (1976). Languages of art: An approach to the theory of symbols. Indianapolis: Hackett.

Granrud, C. E., HaAke, R. J., \& Yonas, A. (1985). Infants' sensitivity to familiar size: The effect of memory on spatial perception. Perception \& Psychophysics, 37, 459-466.

Granrud, C. E., \& Yonas, A. (1984). Infants' perception of pictorially specified interposition. Journal of Experimental Child Psychology, 37, 500-511.

Granrud, C. E., Yonas, A., \& Opland, E. A. (1985). Infants' sensitivity to the depth cue of shading. Perception \& Psychophysics, 37, 415-419

Granrud, C. E., Yonas, A., \& Pettersen, L. (1984). A comparison of monocular and binocular depth perception in 5- and 7-month-old infants. Journal of Experimental Child Psychology, 38, 19-32.

Granrud, C. E., Yonas, A., Smith, I. M., Arterberry, M. E., GlicksMAN, M. L., \& SORKNES, A. (1984). Infants' sensitivity to accretion and deletion of texture as information for depth at an edge. Child Development, 55, 1630-1636.

Gunderson, V. M., Yonas, A., Sargent, P. L., \& Grant-Webster, K. S. (1993). Infant macaque monkeys respond to pictorial depth. Psychological Science, 4, 93-98.

HoRN, B. K. P. (1975). Obtaining shape from shading information. In P. H. Winston (Ed.), The psychology of computer vision (pp. 115-156). New York: McGraw-Hill.
Hudson, W. (1967). The study of the problem of pictorial perception among unacculturated groups. International Journal of Psychology, 2, 89-107.

Kersten, D., Knill, D. C., Mamassian, P., \& Bülthoff, I. (1996). Illusory motion from shadows. Nature, 379, 31 .

Koenderink, J. J., van Doorn, A. J., \& Kappers, A. M. L. (1992). Surface perception in pictures. Perception \& Psychophysics, 52, 487-496.

Koenderink, J. J., van Doorn, A. J., \& Kappers, A. M. L. (1996). Pictorial surface attitude and local depth comparisons. Perception \& Psychophysics, 58, 163-173.

Sen, M. G., Yonas, A., \& KNILL, D. C. (2001). Development of infants' sensitivity to surface contour information for spatial layout. Perception, 30, 167-176.

WALTZ, D. (1975). Generating semantic descriptions from drawings of scenes with shadows. In P. H. Winston (Ed.), The psychology of computer vision (pp. 19-91). New York: McGraw-Hill.

Woodworth, R. S. (1938). Experimental psychology. New York: Holt. YonAs, A., \& ARTERBERRY, M. E. (1994). Infants perceive spatial structure specified by line junctions. Perception, 23, 1427-1435.

Yonas, A., Cleaves, W., \& Pettersen, L. (1978). Development of sensitivity to pictorial depth. Science, 200, 77-79.

Yonas, A., Goldsmith, L. T., \& Hallstrom, J. L. (1978). Development of sensitivity to information provided by cast shadows in pictures. Perception, 7, 333-341.

Yonas, A., Granrud, C. E., Arterberry, M. E., \& Hanson, B. L. (1986). Infants' distance perception from linear perspective and texture gradients. Infant Behavior \& Development, 9, 247-256.

Yonas, A., Granrud, C. E., \& Pettersen, L. (1985). Infants' sensitivity to relative size information for distance. Developmental Psychology, 21, 161-167.

YonAS, A., \& O'DonnelL, S. L. (1996, April). Infants distinguish shadows from markings. Poster presented at the annual meeting of the Association for Research in Vision and Ophthalmology, Fort Lauderdale, FL.

Yonas, A., Pettersen, L., \& Granrud, C. E. (1982). Infants' sensitivity to familiar size as information for distance. Child Development, 53, 1285-1290.

(Manuscript received October 6, 2004; revision accepted for publication March 30, 2005.) 